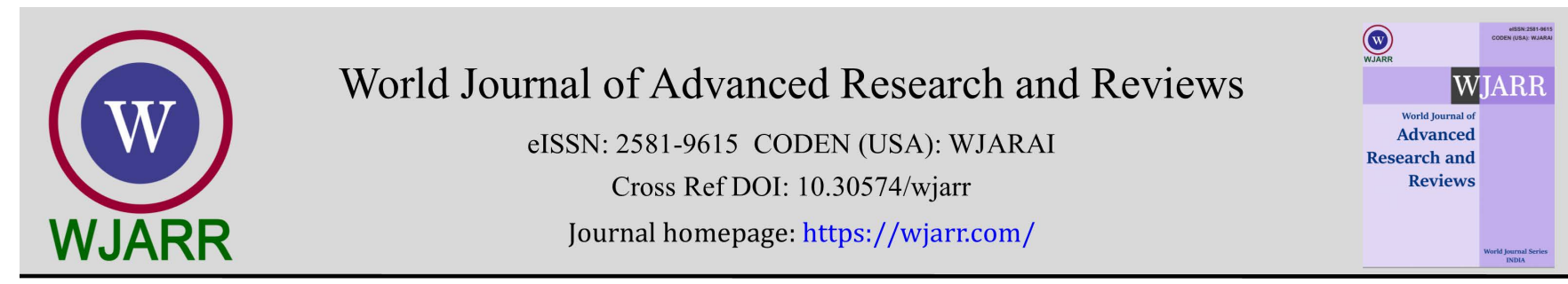

(REVIEW ARTICLE)

\title{
The mass of photons and gravity
}

\author{
Alexander Milanich * \\ Moscow Institute of Physics and Technology (MIPT State University) Moscow, Russia.
}

World Journal of Advanced Research and Reviews, 2021, 09(03), 297-303

Publication history: Received on 13 February 2021; revised on 16 March 2021; accepted on 19 March 2021

Article DOI: https://doi.org/10.30574/wjarr.2021.9.3.0111

\begin{abstract}
This article analyzes concepts of photons and results of old experiments with deflections of light by Sun's gravity. It is possible to draw a conclusion that experimental results differ from theoretical predictions of both classical and General Relativity theories. In this article was proposed explanation based on alternative potential and the mass of photons.
\end{abstract}

Keywords: Mass; Photon; Light deflection; Gravity; Energy

\section{Introduction}

One hundred and two years passed a centenary after first experiments done by Sir Eddington [1], when his team measured the deflection of photons by the Sun's gravity. These results were recognized as experimental proof of the Einstein's General Relativity (or GR) theory validity. This 100-years anniversary is an excellent opportunity to discuss these experiments from the physical and technical point of view and try to understand a degree of reliability of these and other similar experimental results.

These Eddington's experiments in the period of 1919 solar Eclipse [1] became one of the basic experiments of modern physics (testing the basic predictions of GR theory as the background of modern cosmology), but these experiments about never discussed from the point of view its reliability.

Also not many scientists discussed a definition of photons masses today. Let us do it.

\section{Definition of photons masses}

General Relativity (GR) simply postulated that any photon has zero mass and it simply (like a pencil) draw geodesic lines in space. However, this statement causes problems with really existing light pressure. In addition, for photons, it is impossible to measure experimentally the ratio of gravitational to inertial masses, because for inertial mass of photon acceleration ever equal zero due to constant light speed c, and for weighting we need small or zero speed of photons, what is impossible by the same reasons.

Really, we know another one definition of mass $m$ for any types of particles. For photons it is:

$\mathrm{E}=\hbar \omega=\mathrm{mc} 2$

where E - is energy, $\hbar$ - Planck's constant, $\omega$ - frequency of the photon.

\footnotetext{
${ }^{*}$ Corresponding author: Alexander Milanich

Moscow Institute of Physics and Technology (MIPT State University) Moscow, Russia 141701Dolgoprudny, Institutsky per. 9 (Professor, Department of General Physic MIPT Moscow, Russia). 
This definition of photon mass assigned to Louis de Broglie and this concept solved many problems, but this definition and non-zero mass of photons contradicts to A. Einstein GR theory principles.

Because gravity is one from the four fundamental forces, after definition a mass (1) we can calculate deflection of light by gravity in about standard mechanical equations. Before do it, let us analyze real experiments, which were done a century ago.

\section{Accuracy of light deflections experiments}

The Eddington's expedition announced the average displacements of the stars equal to 1.61" + 0.30" (arc seconds) [1]. Almost immediately, it became clear that this accuracy 0.30 " is very good and according to other independent estimations accuracy not exceed 1-2 arc seconds. Hence, it is insufficient for such categorical statements [2] about the validity of GR theory.

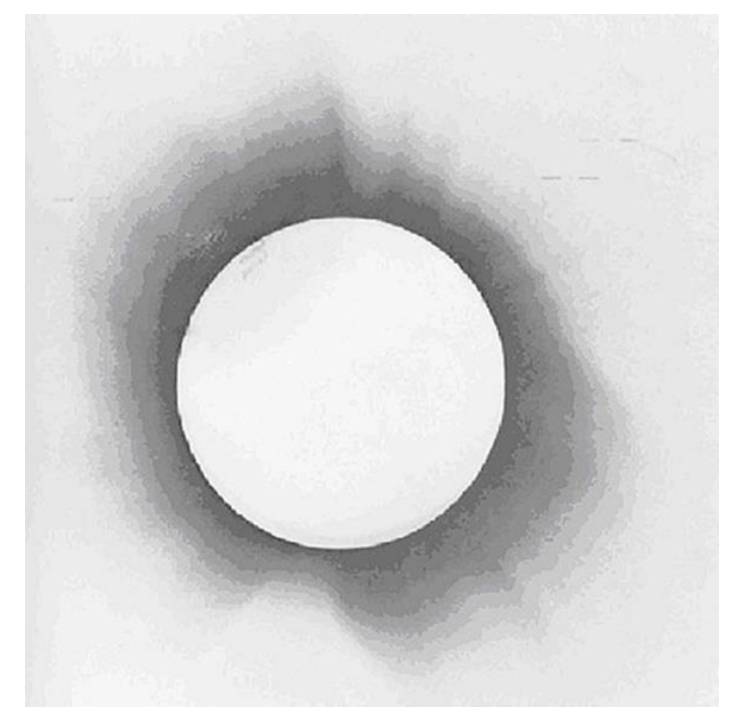

Figure 1 Copy of the original photo of solar Eclipse from an article [1].

Moreover, in original work [1] the authors published several values for the deflection of rays, despite all they were measured in the same expedition and on the same equipment. But on different photographic plates coursed by some unknown reasons they have a different accuracy: 1.98 " +0.12 " and even $2.90 "+0.87$ ". Just a last value of deflection was recognized by the authors as erroneous [1], but without analyzing the reasons of these errors. Such a large variation in accuracy underlines not confidence in the reliability of all results.

According to Einstein's GR theory [2], if light rays pass near the surface of the Sun the deflection should be 1.75". According to J. Soldner [3] or classical calculation, a result should be 2 times smaller - 0.87". By the way, J. Soldner did his calculation in 1804-year and he considers the trajectory of the photon like hyperbola in Newtonian gravity when a photon according to Kepler movement has a constant mass and changes its velocity!

It seems very strange that A. Einstein (who knows his own postulate about constant speed of light), said nothing about incorrect comparison of GR theory angular and Soldner's angular deflection in Kepler's trajectory.

Despite both predicted values are inside interval of measurement's accuracy, Sir Eddington [1] draws a conclusion about the validity of A. Einstein's General Relativity theory. It should be especially emphasized, that any optical experiments with 1"angular accuracy or better are always extremely complicated and especially if they carried out outside the laboratory. The difficulties of experiments with the Sun are especially compounded by the brilliance of the crown (see Fig.1, 2), which prevents the registration of stars near the Sun's surface.

Therefore, in all experiments it was measured deflections of stars images by gravity of the Sun where these stars located at some distance from the Sun's surface (usually more than one Sun's radius). Of cause, it further reduces the predicted value of deflection as $\sim 1,75^{\prime \prime} / \mathrm{R}$ (and $0.87 " / \mathrm{R}$ ) respectively, where $\mathrm{R}$ is the distance from the center of the Sun. 


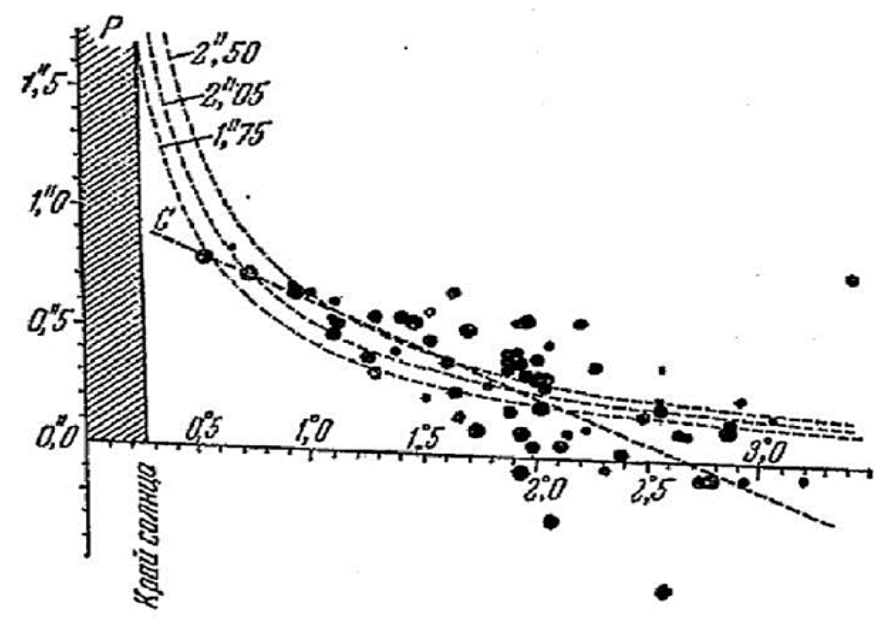

Figure 2 Displacements of the stars [4], where Y (vertical axis) is the angle of deflection in arc seconds, X (horizontal axis) is the distance in degrees from the center of the Sun.

In addition, according to the generally accepted in astronomy method for a more accurate measurement of such small displacements, it is necessary perform control photography of the same sky region (from the same geographical point, on the same equipment, under similar temperature) with an interval near to six months (from the opposite part of the Earth's orbit). Only by comparing the original image of these stars at a moment of solar Eclipse and the control, reference image it is possible to measure deviations of photons with the accuracy better than one arc second.

Fig. 2 represent an example of experimentally obtained results from similar photos (non-orthogonality of axes is due to corrections for changes of scales along the photographic plate).

Without analyzing of other technical details and problems, we note only that Sir Eddington performed control photos from another geographical point (the first at the island Principe but the second at England). Only this fact significantly reduced the degree of confidence of his results.

\section{Alternative results}

Series of similar experiments followed Eddington's expedition between 1919 and 1929 [5]. Although they gave a dispersion of angles displacement of stars at moment of Sun eclipses, in most expeditions, the experimentally determined value of the light deflection near the Sun's surface was essentially greater than predicted by GR theory $1.75^{\prime \prime}$ arc seconds. The average deflections of the light in expeditions 1919, 1922 and 1929 years ranged in the interval between $1.81 "+0.1^{\prime \prime}$ and $2.61 "+0.26^{\prime \prime}[5]$.

It is a very significant systematic error for optical measurements. It has no interpretation and didn't correspond to the effect on the photon from solar coronal plasma, or by the refraction rays in the earth's atmosphere.

Hence, we can draw a conclusion that experimental results differ from both predicted by GR theory 1.75 " and based on wrong classical Newton's model 0.87"!

A. Mikhajlov announced this contradiction in values of experimental deflection for the first time in [5]. He said: "...from results of all observations with a certainty possible to say that the deflection of the light beam passing near the Sun is greater than that calculated by the General Relativity theory...." He said it in 1932!

Other modern experiments (for example, in the radio band) for examination of Einstein's GR theory on the basis of data from radio interferometry of quasars [6] do not cause full confidence yet. This is due to facts that the deflection of radio emission from the solar corona plasma was introduced as a spherical refractive index [7] and it depends on both: the frequency of signals and from the real distribution of electrons in the solar coronal plasma, but it is very difficult to measure correctly. 
Another argument in favor of GR theory, for example, the displacement of the perihelion of Mercury, can be explained by the finite velocity of gravitational waves [8] or by modification of the type of gravitational potential well known today, as post-Newtonian gravity [9] etc.

That is the reason why the interpretation of deflection experiments by gravity is still an urgent task for physicists, both for theorists and for experimenters $[10,11]$.

It was so much attention paid for analyzing of experiments in GR theory, because GR is a background for majority of modern cosmological models. Moreover, we saw that some problems exist yet [10]. Now we'll try to understand, what may be the reason for such significant differences between theories and experiments?

\subsection{Correct calculations of light deflection}

So, comparing of GR theory effects with classical effects calculated by G. Soldner [8] was incorrect, because G. Soldner in 1804 year considers hyperbolic movement of the photon and it was incorrect.

The next remark is about the gravitational constant - G. For GR theory it is the single constant G for any interactions (mass-mass or photon-mass), because gravity will totally define the curvature of space-time in our universe and it depends on a distribution of matter in the universe only. The matter of the universe consists mainly from hadrons (protons and neutrons) and hadrons ever have the same gravitational constant G. However, beyond GR theory, constant $\mathrm{G}$ may differ for photons because it is a boson-hadron interaction. In addition, equivalence of inertial and gravitational masses for photons has no sense, because any photon has the energetical mass only defined by (1).

From 1804 year, sciences accumulated a huge amount of experimental data and many "uncomfortable" questions. For exact solution, the trajectory of a photon and its deflection by the gravity of stars, we need to change our concept of the photon's mass.

Hence, equation (1) introduces a new type of mass. It is not inertial or gravitational; it is energetical mass of the photon, and we will use it in gravitational interactions with hadrons with another (its own) gravitational constant g inside the same Newton gravity law. We remember that a speed of the photon is ever velocity of light c. However, this mass may changes itself in gravitational fields [12], but for a photon, only this energetical mass exists.

In addition, we remember that equivalence for inertial and gravitational masses was experimentally verified for the matter (for hadrons) only, but never for the photon's mass. Moreover, even for matter the equivalence of inertial and gravitational mass (more exactly, the mass ratio) is checked with an accuracy till 10-12, but it was recorded small changes in the gravitational constant [11] (or in mass?), which still do not have an acceptable explanation. In addition, no one knows there is or not a dependence of the gravitational constant, for example, from the isotopic composition of matter? Also, the gravity was never experimentally measured for leptons, despite that is fundamental force and so on.

In principle, Sir Edington's experiments were one of the first experiments for measurements gravity between hadrons and bosons (photons) and by changing gravitational constant G we may explain any experimental result for light deflections.

In article [13] it was shown that for photons in gravitational fields it is possible to use a potential (2). However, with another, new g (gravitational constant). This new constant g corresponds for photons-hadron (or boson-hadron) interaction.

$$
\hbar \omega=\hbar \omega 0 \exp (\mathrm{Rs} / 2 \mathrm{R})
$$

where Rs - is Szhwartzshild radius, as some gravity parameter, $\omega 0$ - is a frequency at infinity and R is a distance from the gravity center.

No problem exists to modify classical theory for light deflection [4] by changing value of $\mathrm{G}$ to $\mathrm{g}$, but it is impossible to save GR theory, because any values differ from 1.75 " arc seconds contradict to principals of GR theory.

Note that the other potential for photons was introduced in 1994 in article [13] too, but presented in [13] potential had an inverse dependence from $\mathrm{R}$, what was due to incorrect transfer the ideas about the mechanical potential (with fixed masses) on the concept of photons with variable mass. 
From the experimental fact that the measured deflection of photons by the Sun exceeds two angular seconds, it is possible to draw a conclusion that the gravitational constant $\mathrm{g}$ for the photon is about 2.4 times more than $\mathrm{G}$ the standard gravitational constant for matter (for hadrons), as it was calculated in paragraph 4.2.

\subsection{Calculations of photon trajectory}

Hence, the classical calculation of the deflection angle for photons by J. Soldner was erroneous. Taking into account the new gravitational constant for the photon and the changeable mass of photons in the gravitational field, it turned out that a new approach used for correct calculations of photon trajectory. It based on the law of conservation of angular momentum in the central gravitational field and principal statement about fixed speed of light. The validity of the law of conservation of the amount of angular momentum follows from space isotropy according to E. Noether theorem [14].

To simplify the calculation, let us choose a coordinate system so that its origin is the center of the Sun, and the photon moves from minus infinity parallel to the x- axis. Let us neglect by contribution in trajectory expansion of the universe, and multiplying the left and right parts of (2) by the impact parameter a. The conservation for angular momentum in such coordinate system has the following simple form:

$\hbar \omega \mathrm{a}(\mathrm{x}, \mathrm{y})=\mathrm{const}=\hbar \omega 0 \mathrm{a} 0$

here we used all previous symbols, and $\mathrm{a} 0$ and $\mathrm{a}(\mathrm{x}, \mathrm{y})$ - impact parameters at the beginning a 0 and in coordinates $\mathrm{x}, \mathrm{y}$.

As it follows from equation (3), the photons no longer moves along hyperbolas, although its trajectory can always be extrapolated by some abstract hyperbole at large distances from the Sun. Now the mass of the photon increases when it approaches the Sun and it depends only on the distance to the Sun's center.

The impact parameter decreases and the photon's vector of velocity rotated so that the law of conservation of angular momentum was fixed.

We are unable to find the exact analytical solution for the photon trajectory in the range $\mathrm{x}$ - from infinity to zero, but there is no problem to calculate parameters of its trajectory by computer. To do this, let us determine the nearest (minimal) distance $\mathrm{d}$ between the photon and the Sun. After this point (minimal distance), the photon begins to lose its mass, and the impact parameter begins to increase.

From equation (3) we'll find:

$\mathrm{a}(\mathrm{x}, \mathrm{y}) \mathrm{m}(\mathrm{x}, \mathrm{y})=\mathrm{d} \mathrm{m} 0 \exp (\mathrm{Rs} / 2 \mathrm{~d})=(\mathrm{a} 0+\mathrm{d}) \mathrm{m} 0$

where $\mathrm{m} 0$ and $\mathrm{m}(\mathrm{x}, \mathrm{y})$ - the masses of the photon at different (sighting) distance from the Sun.

Near the Sun, because the radius of the Sun d is 699 thousand $\mathrm{km}$, for the new gravitational constant g we will obtain Rs $=\mathrm{gM} / \mathrm{c} 2=4.25 \mathrm{~km}$, and it does not depends on the energy (wavelength) of photons. Decomposing equation (4) into Taylor series, we obtain that the minimum sighting distance near the surface of the Sun will be on $4.25 \mathrm{~km}$ less than the impact parameter at infinity.

In the expanded form, the law of conservation of angular momentum determines the exact trajectory of the photon's motion. In the coordinate system $Y, X$ coordinates with the origin in the center of the Sun, for the photon at the beginning its movement from minus infinity parallel to the axis $X$ at the initial impact parameter (at a distance a0), the correct equation of the trajectory of the photon takes the form:

$\mathrm{a} 0=(\mathrm{y}-\mathrm{x}(\mathrm{dy} / \mathrm{dx})) \exp (\mathrm{Rs} / 2 \mathrm{R}) /\left(1+(\mathrm{dy} / \mathrm{dx})^{\wedge} 2\right)$

where $R(x, y)=\sqrt{ }\left(x^{\wedge} 2+y^{\wedge} 2\right)$

In addition to the previously defined variables, here $\mathrm{x}$ and $\mathrm{y}$ are the coordinates of the photon and $\mathrm{R}$ is the distance from the photon to the center of gravity (to the center of the Sun). *We consider a photon like a point in this classical iteration now.

In fact, the equation (5) records the distance from the origin (the center of the Sun) to the line passing from the point $(\mathrm{x}, \mathrm{y})$ at a given angle $\mathrm{dy} / \mathrm{dx}$, and this is the impact parameter for an arbitrary point $(\mathrm{x}, \mathrm{y})$. By multiplying the impact 
parameter at some point and the mass of the photon at the same point it is possible to perform the conservation law for angular momentum (5).

It means that the quasi-classical approximation was used, when the photon is considered as a point of variable mass with coordinates $(\mathrm{x}, \mathrm{y})$ and with a fixed velocity $\mathrm{c}$.

As we said above, unfortunately, this photon trajectory has no analytical solution, but the system of equations (5) easily solved at simplest computers (see Fig.3).

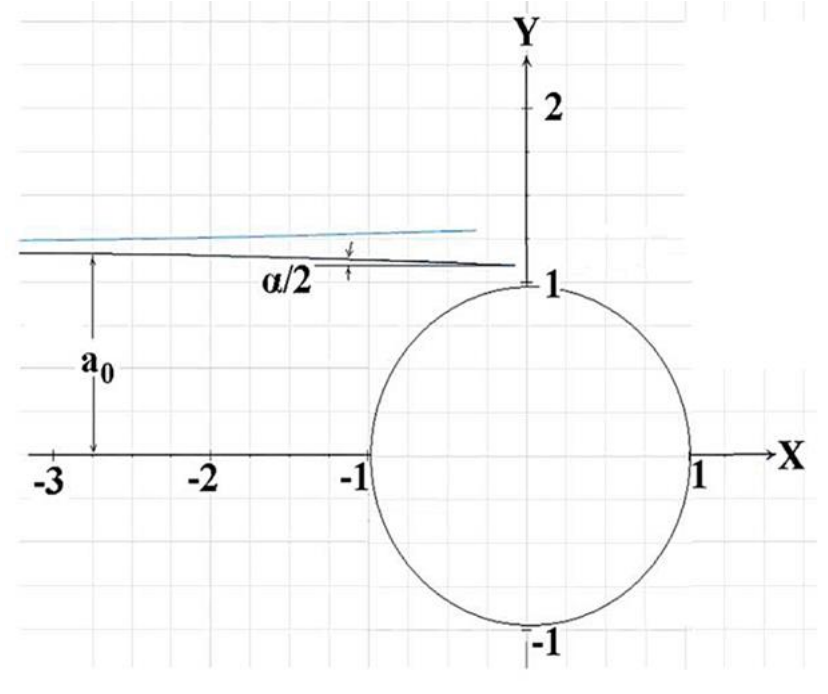

Figure 3 Trajectory for photons in the gravity of the Sun.

The unit along the $\mathrm{X}$ and $\mathrm{Y}$ axes corresponds to the radius of the Sun, the parameter $\mathrm{Rs}=0.1$, and a0 $=1.2$

Although the solution of the system (5) is ambiguous (due to different signs of the roots from the square of the derivative) and is valid only for negative $\mathrm{x}$ (because it does not take into account the photon deviation for positive $\mathrm{x}$ ), but supplemented by the physical sense of the system (5) allow easy to choose the only correct solution (the lower trajectory at Fig.3).

Of cause, the scales at Fig.3 was enlarged, because otherwise arc seconds degrees would be invisible.

\section{Conclusion}

Therefore, it can argue that both predicted by GR theory light deflection values (1.75") and "classical" deflection ( 0.87 ") do not correspond to experiments. Consequently, experimental deflection of photons by solar gravity has dealt a serious blow not only to classical theory of Newton's gravity but to GR theory too! Only deflection in 1.75" approves GR theory, but many experiments said that it is more than 2" arc seconds are. It means that we need more careful experiments in light deflection or frequency deviations in gravitational fields to be sure (or not) in GR theory.

Also, for photons with changeable mass, we need another potential. This new potential has saved photon's characteristics, for example, the energy accumulated by photons itself, and having ability to change its mass (energy), the photon always leaves the "black hole" [6], it demonstrates the properties of lensing, localization inside "black holes" or even overcome Schwarzschild's sphere.

After expansion in Taylor series and an approximation for small angles of deflection $\alpha$ we see:

$\alpha \sim 2 \mathrm{k}(\mathrm{d})=2 \mathrm{RS} / \mathrm{R}=7,08 / 69910-3 \sim 10.12 * 10-6=2.09 "$

Hence, this new concept of photon and gravity can explain real experimental results of light deflection (6). New concepts and new potential radically change the concept of black holes too, because now photons ever go away from any gravitational center, etc. In any cases, we need new experiments. 


\section{Compliance with ethical standards}

\section{Acknowledgments}

Many thanks to my colleges for support of this work.

\section{References}

[1] Dyson FW, Eddington AS, Davidson CA. Determination of the Deflection of Light by the Sun's Gravitational Field, from Observations Made at the Total Eclipse of May 19, 1919 // Phil. Trans. Roy. Soc. of London. Ser. A, 1920; 220: 291-333.

[2] Brian G. The Elegant Universe // M: Published USSR; 2004; 59.

[3] Soldner JG. Ueber die Ablenkung eines Lichtstrahls von seiner geradlinigen Bewegung, durch die Attraktion eines Weltkörpers, an welchem er nahe vorbei geht // Berliner Astronomisches Jahrbuch. 1804; 161-172.

[4] Mikhailov AA. Observation of the Einstein effect during solar eclipses // UFN. 1956; 59(1): 51-66.

[5] Kushnir Yu M, Fursov VS. New observations of the deflection of light rays in the Sun's gravity field. // UFN 1932. v. 12(1). P.159. 2th ed. New York: McGraw-Hill. 2011; 1505-20.

[6] Muhleman DG, Ekers RD. Fomalont EB. Radio Interferometer Test of the General Relativistic Light Bending Near the Sun // Phys. Rev. Lett. 1970; 24(24); 1377-1380.

[7] Tsupko O.Yu., Bisnovatyi-Kogan G.S. Gravitational lensing in plasma: Relativistic images at homogeneous plasma // Physical Review D. 87. 2013. 124009: 1- 16.

[8] Gerber P. Die räumliche und zeitliche Ausbreitung der Gravitation // Z. Math. Phys. 1898; 43: 93-104.

[9] Epstein R., Shapiro Irwin I. Post-post-Newtonian deflection of light by the Sun // Phys. Rev. D. 1980; 22(12): 2947-2949.

[10] Parra JL. Photonic Gravitational Interactions from a Quantum Point of View // Optics and Photonics Journal. 11: $12-21$.

[11] Karagioz OV. Theoretical and experimental studies of torsional weights and systems based on them for measuring dissipative processes, gravitational and pondemotor interactions // Abstract dissertation for the degree of Doctor of technical sciences. M. 1998; 23.

[12] Pound RV, Rebka JrGA. Apparent weight of photons // Physical Review Letters. 1960; 4(7): 337-341.

[13] Troitsky VS. Experimental evidence against the Big Bang cosmology // UFN. 1995; 165(6): 706.

[14] Noether E. Invariant Variation Problems // Available from arXiv: 8 Mar 2005 - physics [physics.hist-ph] / 0503066 - v1

[15] Romanko VN, Prokopov AV, Matvienko SA, Romanko OV. State and prospects of development of the radiophysical method for measuring the gravitational potential of the Earth // Kharkov, Metrology. 2012; 152-155. 